\section{Estimating sample properties of principal components using SAS}

\author{
A. NARAYANAN \\ Indiana University, Bloomington, Indiana
}

An SAS (statistical analysis system) program for computing the standard errors of principal component coefficients (obtained using the covariance matrix) is presented. The standard errors are useful for checking the stability of the coefficients, which pre vents the user from overinterpreting the results. An application of the usefulness of the program is illustrated through an example.

Principal component analysis is a multivariate technique for summarizing information in a set of interrelated variables. This is achieved by transforming the original variables into uncorrelated variables by taking linear combinations that maximize variance. Geometrically, the new variables, called principal components, are orthogonal axes constructed in the direction of maximum variances. A measure of the amount of information in each component is given by its variance, and the first few components arranged in the order of decreasing variance typically summarize most of the information in the data.

Principal component analysis is used extensively in psychological applications to discover the underlying structure of data. In the original application of the technique, Hotelling (1933) showed that there are two major components to responses on entry-level examination tests: verbal and quantitative ability. In this context, the principal components are interpreted by using the component coefficients as "loadings." This interpretation can sometimes be dangerous when the coefficients are unstable, and it can lead to conclusions different from the true structure of the data. In this paper, we remind readers that standard errors of the coefficients are useful quantities to look at, and we provide a statistical analysis system (SAS) program that will compute these as part of a principal component analysis.

Few textbooks on applied multivariate analysis emphasize the sample properties of principal components, and even among those that do, the topic is relegated to a less important final section of the chapter. However, the standard errors of principal components contain valuable information when the coefficients are used for interpretation (Flury \& Riedwyl, 1988). These standard errors can be used as the standard errors in regression analysis are used, so that the user will not overinterpret the results. To quote Jackson (1991): "This is a quantity that needs to be ex-

Correspondence should be addressed to $A$. Narayanan, Department of Decision and Information Systems, Graduate School of Business, Indiana University, Bloomington, IN 47405. ploited. We are sophisticated enough to quote standard error of a regression coefficient along with the coefficient itself; why not do the same for characteristic vectors?"

To interpret the coefficients meaningfully, we need to assume that the data are a sample from a multivariate normal population with a specified covariance structure. In what follows, the notation is similar to that of Morrison (1990); the boldface represents the appropriate vectors or matrices. Let $x_{1}, x_{2}, \ldots, x_{n}$, be $n$ independent $p$ dimensional random variables with an $N_{p}(\mu, \Sigma)$ distribution. Suppose that $\boldsymbol{\Sigma}$ has distinct characteristic roots,

$$
\lambda_{1}>\lambda_{2} \ldots \lambda_{p}>0,
$$

with corresponding eigenvectors

$$
\alpha_{1}, \alpha_{2}, \ldots, \alpha_{p}
$$

Denote the sample estimate of $\mathbf{\Sigma}$ by $\mathbf{S}$, and let the sample estimates of eigenvalues and eigenvectors of $\mathbf{S}$ be denoted by

$$
l_{1}>l_{2}>\ldots l_{p}
$$

and

$$
\mathbf{a}_{1}, \mathbf{a}_{2}, \ldots, \mathbf{a}_{p}
$$

Girshick (1939) and Anderson (1963) have shown that the following results hold as $n$ becomes large:

1. $l_{i}$ is distributed independently of the elements of $a_{i}$.

2. $\sqrt{n}\left(l_{i}-\lambda_{i}\right)$ is asymptotically normally distributed with mean zero and variance $2 \lambda_{i}^{2}$, independently of other eigenvalues.

3. $\sqrt{n}\left(a_{i}-\alpha_{i}\right)$ is distributed according to a multivariate normal distribution, with mean vector zero and covariance matrix

$$
\lambda_{i}=\sum_{\substack{h=1 \\ h \neq i}}^{p} \frac{\lambda_{h}}{\left(\lambda_{h}-\lambda_{i}\right)^{2}} \alpha_{h} \alpha_{h}^{\prime} .
$$

In the sample, the standard error $(S E)$ of the $j$ th component of the $h$ th eigenvector is computed as

$$
S E\left(a_{h j}\right)=\left[\frac{1}{n} l_{h} \sum_{\substack{k=1 \\ k \neq h}}^{p} \frac{l_{k}}{\left(l_{k}-l_{h}\right)} a_{k j}\right]^{1 / 2} .
$$

Although the computation is straightforward, it is quite laborious to calculate on a pocket calculator. We recommend that these statistics be computed as part of every principal component analysis and offer a simple SAS (SAS Institute, $1990 \mathrm{a}, 1990 \mathrm{~b}$ ) program for that purpose.

It should be noted that the results are asymptotic and are valid only under the conditions that the covariance matrix is positive definite and that the eigenvalues are distinct. The latter condition is especially critical, since the eigenvectors are not uniquely defined if the corresponding eigenvalues are not distinct. This also has implica- 
tions for the standard error. From the formula above, we note that any pair of eigenvalues being close (numerically) would inflate the standard error. In the example given later (see Appendix B), the fourth and the fifth smallest eigenvalues are numerically similar, which inflates the standard errors of the corresponding eigenvectors (PRIN4 and PRIN5).

It should also be noted that the results given here are applicable for raw principal components. Although "standard" principal component analysis does not include rotation (one of the main ideas of factor analysis), there may be circumstances in which rotation may be advantageous. In such cases, care must be employed in using these results. If the rotation is rigid (e.g., varimax), the resulting components will also be normally distributed, because this is a linear transformation and the mean and the variance will correspond to this transformation. For other types of rotation, these results are not easily extendable.

\section{Comments and Instructions}

An example of an SAS program using the SAS/IML software (SAS Institute, 1990a) is presented in Appendix A. The program is presented along with the SAS procedure PROC PRINCOMP, which is used in computing the principal components. The user should be aware that the results in this paper are valid for analysis based on the covariance matrix, and that the option COV should be used in the PROC PRINCOMP statement. It is also assumed that the analysis is based on the covariance matrix corrected for the means. If the uncorrected matrix is used for the analysis, the NOINT option will allow the program to be used with obvious changes in the interpretation. The program uses the dataset created from PROC PRINCOMP with the OUTSTAT = option and reads the appropriate rows of the data into vectors and matrices. See the SAS user's guide (SAS Institute, 1990b) for details.

\section{Example and Verification}

In illustrating the program, we use the banknote example of Flury and Riedwyl (1988). The data correspond to measurements on six characteristics of genuine and forged 1,000-franc bills. Only the data (100 observations) corresponding to genuine bills are used here for illustration. The six characteristics measured are $X_{1}$, length of bill; $X_{2}$, width of bill measured on the left; $X_{3}$, width of bill measured on the right; $X_{4}$ width of margin at the bottom; $X_{3}$, width of margin at the top; and $X_{6}$, length of image diagonal. The data are input; the SAS procedure PROC PRINCOMP is invoked; and the results are obtained in an SAS data set by using the OUTSTAT = option. The principal component coefficients, shown in Appendix $B$ (they should be read columnwise), match with Table 10.1 of Flury and Riedwyl (1988). The standard error of principal component coefficients is shown later; these values match with Table 10.5 in Flury and Ried- wyl. From the eigenvalues, it is clear that the first three components explain $85.5 \%$ of the total variance, and the first four components, $91.4 \%$.

The stability of the coefficients can be seen by looking at the standard error matrix (SB) or the $z$-ratio matrix (ZR), where each element is a ratio of the coefficient to its standard error. By using a rule of thumb of choosing coefficients whose absolute " $z$ value" exceeds 2 , we see that, in the first component (PRIN1), only variables $X_{4}$ and $X_{5}$ are stable. Similarly, Component 2 looks reasonably stable in all the variables, and coefficients of Components 4 and 5 look quite unstable, because they are estimated with large standard errors. Without consideration of the standard errors, there may be a temptation to interpret these components. This may also be an indication of when to stop "extracting" components, which is one of the difficult problems in principal component analysis.

In trying to interpret the components, we can use the coefficients given in the principal component coefficient matrix. Using these coefficients, we see that the first component is essentially a difference between $X_{4}$ (TOP) and $X_{5}$ (BOTTOM). According to Flury and Riedwyl (1988), there is a possible explanation for this: perhaps the bills were cut after the image had been printed on large pieces of paper and the cutting mechanism was rather inaccurate. By the same rule, Component 2 is a weighted average of all the variables. Such a component can be considered as a "size" component, because large values of this component indicate a large bill. Component 3 does not have an obvious interpretation.

In this paper, we have presented a program for computing standard errors of principal component coefficients and illustrated it with an example. We recommend that these be computed as part of routine analysis, so that the user will not overinterpret the results, and we suggest that programs built in the future carry options to compute these quantities.

\section{REFERENCES}

ANDerson, T. W. (1963). Asymptotic theory for principal component analysis. Annals of Mathematical Statistics, 34, 122-148.

FlURY, B., RIEDWYL, H. (1988). Multivariate statistics: A practical approach. London: Chapman \& Hall.

Girshick, M. A. (1939). On the sampling theory of roots of determinantal equations. Annals of Mathematical Statistics, 10, 203-224.

Hotelung, H. (1933). Analysis of a complex of statistical variables into principal components. Journal of Educational Psychology, 24, 417-441.

JACKSON, E. (1991). A user's guide to principal components. New York: Wiley.

MoRkson, D. G. (1990). Multivariate statistical methods (2nd ed.). New York: McGraw Hill.

SAS INSTTIUTE (1990a). SAS/IML user's guide (Version 6 edition). Cary, NC: Author

SAS INSTITUTE (1990b). SAS user's guide (Version 6 edition). Cary, NC: Author. 


\section{APPENDIX A}

SAS Program for Principal Component Analysis That Will Also Calculate the Standard Errors of PC Coefficients (Anderson, 1963)

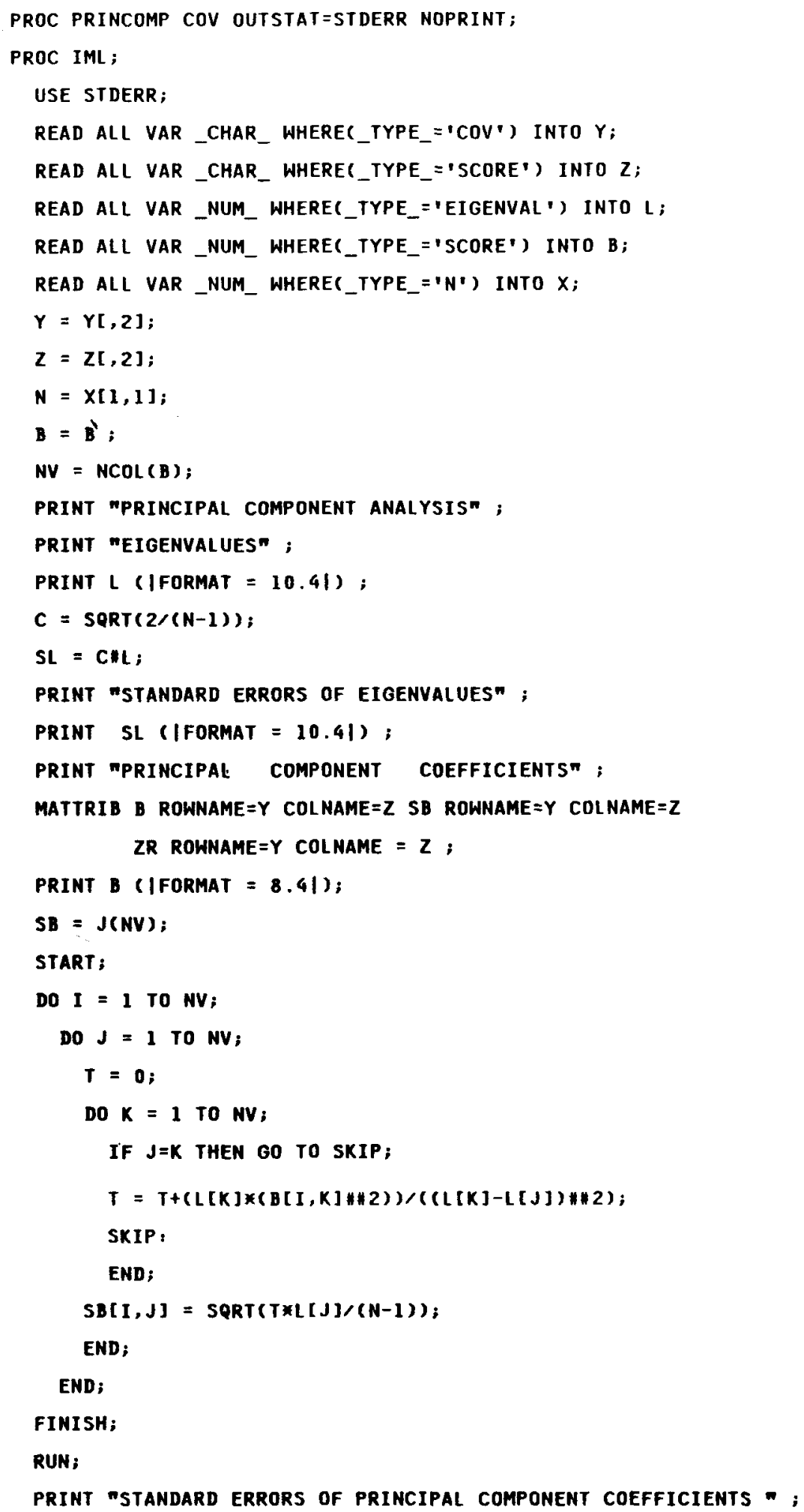


PRINT SB $(\mid$ FORMAT $=8.4 \mid) ;$

$Z R=B / 5 B$;

PRINT "Z - RATIO MATRIX" ;

PRINT ZR (|FORMAT $=8.41)$;

APPENDIX B

Principal Component Analysis

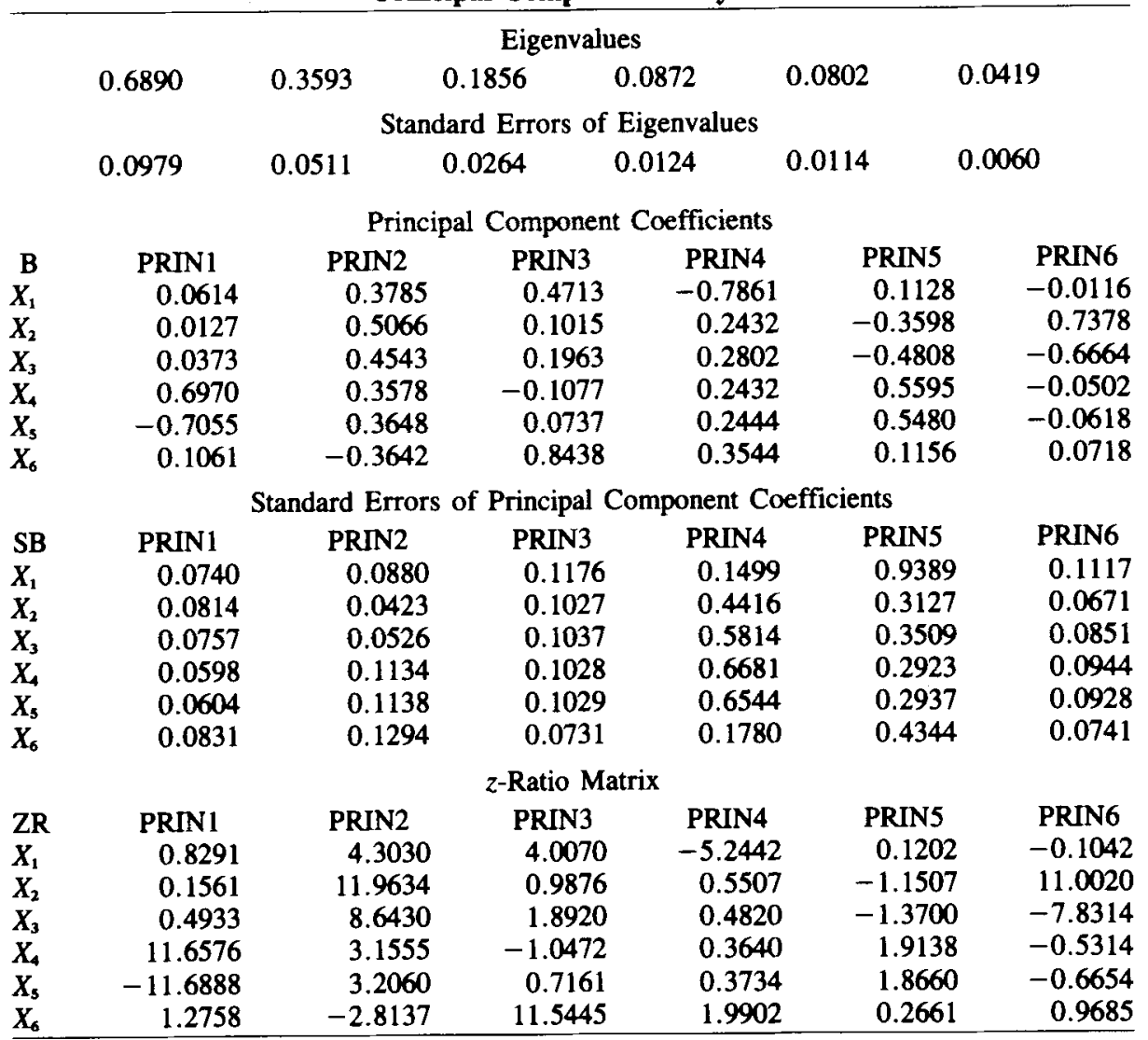

(Manuscript received April 22, 1991;

revision accepted for publication July 22,1991 .) 